\title{
Electroacupuncture in fibromyalgia: results of a controlled trial
}

\author{
Christophe Deluze, Lorenzo Bosia, Angelica Zirbs, Alex Chantraine, Thomas L Vischer
}

\begin{abstract}
Objective-To determine the efficacy of electroacupuncture in patients with fibromyalgia, a syndrome of unknown origin causing diffuse musculoskeletal pain.

Design-Three weeks' randomised study with blinded patients and evaluating physician.

Setting-University divisions of physical medicine and rehabilitation and rheumatology, Geneva.

Patients -70 patients (54 women) referred to the division for fibromyalgia as defined by the American College of Rheumatology.

Interventions-Patients were randomised to electroacupuncture $(n=36)$ or a sham procedure $(n=34)$ by means of an electronic numbers generator.

Main outcome measures-Pain threshold, number of analgesic tablets used, regional pain score, pain recorded on visual analogue scale, sleep quality, morning stiffness, and patient's and evaluating physician's appreciation.
\end{abstract}

Results-Seven of the eight outcome parameters showed a significant improvement in the active treatment group whereas none were improved in the sham treatment group. Differences between the groups were significant for five of the eight outcome measures after treatment.

Conclusions-Electroacupuncture is effective in relieving symptoms of fibromyalgia. Its potential in long term management should now be studied.

\section{Introduction}

Patients with fibromyalgia or the fibrositis syndrome suffer from diffuse musculoskeletal pain and constitutional symptoms such as fatigue, disturbed sleep, vertigo, and abdominal discomfort. ${ }^{1}$ Diagnosis is established by the detection of the characteristic tender points. Diagnostic criteria have been proposed by several authors. Aetiology and pathogenesis remain unclear despite extensive research. There is no specific treatment, and the different approaches to management often give disappointing results both for patients and for physicians. ${ }^{2}$ Many patients therefore try alternative medicine. Acupuncture may be counted as an alternative medicine.

Objective physiological effects of acupuncture have been recorded when the traditional needles are manipulated after insertion or connected to an electric current. In animals electroacupuncture inhibits nociceptive inputs at several levels of the central nervous system. ${ }^{34}$ In humans electroacupuncture raised the pain threshold in dental pain, ${ }^{5}$ experimental heat pain, ${ }^{6}$ and painful nerve stimulation. ${ }^{7}$ Despite these data the efficacy of acupuncture in osteoarticular pain remains controversial, ${ }^{8}$ mainly because of inappropriate methodology in clinical trials. To our knowledge there have been only two studies in primary fibromyalgia ${ }^{10}$; one was retrospective and neither was randomised. We report a prospective, randomised, blind study aimed at seeing whether electroacupuncture has an objective effect in fibromyalgia.

\section{Patients and methods}

Adult patients referred to our divisions for fibromyalgia as defined by the American College of Rheumatology ${ }^{11}$ (widespread pain, mild or greater tenderness in 11 or more of 18 tender point sites) were admitted to the study. Exclusion criteria were severe concomitant disease, treatment with morphine-like drugs or anticoagulants, peripheral neuropathy, bleeding disorders, language difficulties, and past treatment with acupuncture. The patients continued with their other usual treatments (physiotherapy, analgesics, anti-inflammatory agents, tricyclic antidepressants). After recruitment the patients recorded the number of tablets taken for pain during the last week before beginning the study.

DESIGN

The study was approved by the department of medicine's ethics committee.

After recruitment and before giving informed written consent patients were told that the study was to see if electroacupuncture was effective in fibromyalgia, electroacupuncture being still experimental. The treatment procedure would consist of introducing needles into the skin with subsequent stimulation with electric current. Two different methods of electroacupuncture would be compared, patients being allocated one or other treatment by chance. Patients were then randomised to electroacupuncture or control. Randomisation was by means of an electronic numbers generator. Seventy closed envelopes, numbered 1-70, were prepared before the study and opened in numerical order after recruitment of each patient.

Treatment consisted of six sessions of electroacupuncture spread over three weeks. An electrostimulator (Unipuls, Seirin AG, Neu-Isenburg, Germany) with five pairs of electrodes was used. The current was rectangular with a biphasic top out voltage of 10 volts at $1000 \mathrm{ohm}$ and frequency $1-99 \mathrm{~Hz}$ with continuous scanning of the frequency spectrum; every $250 \mathrm{~ms}$ an interval of $250 \mathrm{~ms}$ was programmed. Intensity of the current was maximally $10 \mathrm{~mA}$, which is above the perception threshold but just below the pain threshold and induces a visible muscular contraction. Four to 10 stainless steel needles $(0.3 \mathrm{~mm}$ by $25 \mathrm{~mm}$, excluding the handle), autoclaved before use, were implanted to a depth of 10-25 mm and fixed with tape. Depth of insertion was determined according to the sensitivity of the site ("needling sensation") as indicated by the patient. ${ }^{12}$ In patients having electroacupuncture four common acupuncture points were used-the first dorsal interosseous muscle of the hand and the anterior tibial muscle $(5 \mathrm{~cm}$ beneath the inferior margin of the patella and $1 \mathrm{~cm}$ below the anterior crest of the tibia) on both sides. At most six other sites were chosen 
depending on the patient's symptoms and pain pattern and according to the empirical efficacy of the sites in the treatment of pain. ${ }^{13}$

In the controls a similar number of needles were used but they were put about $20 \mathrm{~mm}$ away from the point which would have been chosen for real electroacupuncture, including the four points common to all patients. The needles were inserted to a depth of 3-4 $\mathrm{mm}$ and fixed with tape. The current used was similar to but weaker than that used in the real procedure. No increase was made after the threshold of perception had been reached. There was no muscular contraction. Both electroacupuncture and sham electroacupuncture were done by the same investigator (CD). Patients were seen individually, at different times, and had no opportunity to meet.

\section{EVALUATION}

The evaluating physician, who was unaware of which treatment group the patients were in, was the same for each patient. Evaluation was done before the first session and after the treatment and comprised eight measurements: pain threshold, number of analgesic tablets used, regional pain score, pain recorded on visual analogue scale, sleep quality, morning stiffness, and patient's and evaluating physician's appreciation of the patient's general state.

Pain threshold was determined by a pressure gauge (PTH-AF2, Pain Diagnostics and Thermography Corporation, Greatneck, New York), ${ }^{14}$ calibrated in $\mathrm{kg} / \mathrm{cm}^{2}$, attached to a plunger with a $1 \mathrm{~cm}$ round rubber tip. It is used to assess sensitivity by applying constantly increasing pressure at a rate of $1 \mathrm{~kg} / \mathrm{cm}^{2} / \mathrm{s}$ over the tender points until the subject first feels pressure change to pain. Pain threshold was measured over 18 tender points as defined by the American College of Rheumatology ${ }^{11}$ and the area with the lowest value used for the evaluation. Use of analgesic tablets was measured as the number of tablets used during the last week before evaluation. Regional pain score was derived by means of a body drawing on which 21 regions were indicated. The patient assessed the pain in each region on a scale of $1-5$, where 5 was worst. Patients also assessed pain by using a visual analogue scale $(1-100 \mathrm{~mm})$. Sleep quality was measured on a scale of $1-10$, where 10 was best. Morning stiffness was measured in minutes. Patients scored their own general state on a scale of $1-10$, where 10 was best. The evaluating physician's impression of the patient's general state was scored on a similar scale.

STATISTICAL ANAI.YSIS

Based on the study by Lautenschläger et al, where

TABLE I-Characteristics of patients. Mean values expressed with (SE) and [95\% confidence interval]

\begin{tabular}{lcc}
\hline & $\begin{array}{c}\text { Controls } \\
(\mathrm{n}=34)\end{array}$ & $\begin{array}{c}\text { Acupuncture } \\
(\mathrm{n}=36)\end{array}$ \\
\hline No of women/No of men & $21 / 13$ & $33 / 3^{\star}$ \\
Mean age (years) & $49 \cdot 0(2 \cdot 0)[44 \cdot 8$ to $53 \cdot 1]$ & $46 \cdot 8(2 \cdot 3)[42 \cdot 2$ to $51 \cdot 3]$ \\
Mean duration of disease (years) & $6 \cdot 9(1 \cdot 3)[4 \cdot 3$ to $9 \cdot 6]$ & $14 \cdot 4(3 \cdot 7)[6 \cdot 9$ to $22 \cdot 0]$ \\
Mean severity of disease (scale $1-5) \dagger$ & $3 \cdot 0(0 \cdot 1)[2 \cdot 7$ to $3 \cdot 3]$ & $2 \cdot 8(0 \cdot 1)[2 \cdot 6$ to $3 \cdot 0]$ \\
\hline
\end{tabular}

${ }^{\star} \mathrm{p}=0.015\left(\chi^{2}\right.$ test $)$. $+1=$ Best.

TABLE II-Clinical parameters. Values are means (SE) [95\% confidence interval] pain threshold as assessed by pressure gauge differed by $0.35 \mathrm{~kg} / \mathrm{cm}^{2}$ between the group treated with acupuncture and the control group, ${ }^{10}$ we calculated that we would need 68 patients for our study $(\alpha=5 \%$, $\beta=20 \%$ ). As some of the scales were not symmetric non-parametric tests were used for analysis. For the calculation of intergroup differences the MannWhitney $U$ and Wilcoxon rank sum W test (two tailed) was used. Intragroup differences were calculated by the Wilcoxon matched pairs signed ranks test (two tailed).

\section{Results}

Seventy patients entered the study. Thirty four were randomised to the control group and 36 to the electroacupuncture group. The two groups were not significantly different in any aspect except for an excess of men in the control group ( $p=0.015)$ (tables I and II). Fifteen patients withdrew from the study and were not re-evaluated. In the electroacupuncture group six patients withdrew for reasons associated with the procedure (increase in symptoms in two patients, unpleasantness of needle insertion in three, ankle oedema in one), one did not return, and one patient was hospitalised for an unrelated condition. In the control group five patients withdrew for reasons associated with the procedure (increase in symptoms in four, unpleasantness of needle insertion in one) and two were hospitalised for other conditions. The 15 patients who withdrew did not differ significantly at the beginning in any parameter from the whole population (data not shown).

The results are given in table II. Patients in the electroacupuncture group improved significantly in all parameters except morning stiffness whereas there was no change in the controls (tables II and III). Posttreatment values were significantly better after electroacupuncture in five of the eight parameters studied when compared with the control group (table III). Pain threshold, which we considered to be the main parameter, improved by $70 \%$ in the electroacupuncture group and $4 \%$ in the control group. This difference was significant.

The overall results after electroacupuncture were that about half of the patients improved satisfactorily whereas a quarter had no change in symptoms. The remainder showed an unexpectedly large improvement with almost complete disappearance of symptoms. They seemed not to differ from the other patients in severity and duration of symptoms or in responsiveness to previous treatments. This degree of improvement was observed in only one case in the control group.

Because of the practical limits of the study patients were not evaluated further.

\section{Discussion}

In this study patients treated with electroacupuncture experienced greater improvement than the controls. Evaluating treatment in fibromyalgia is

\begin{tabular}{|c|c|c|c|c|}
\hline & \multicolumn{2}{|c|}{ Before treatment } & \multicolumn{2}{|c|}{ After treatment } \\
\hline & Control $(n=27)$ & Acupuncture $(n=28)$ & Control $(n=27)$ & Acupuncture $(n=28)$ \\
\hline Pain threshold $\left(\mathrm{kg} / \mathrm{cm}^{2}\right)$ & $1.47(0.24)[0.97$ to 1.98$]$ & $1.36(0.21)[0.94$ to 1.79$]$ & $1.54(0.23)[1.07$ to 2.01$]$ & $2.32(0.32)[1.67$ to 2.98$]$ \\
\hline No of analgesic tablets during last week & $7.93(1.78)[4.27$ to 11.59$]$ & $10.36(2.97)[4.25$ to 16.47$]$ & $10.07(2.17)[5.61$ to 14.54$]$ & $6.86(2.84)[1.04$ to 12.68$]$ \\
\hline Regional pain score $\left(1^{\star}-105\right)$ & $36.96(2.53)[31.75$ to 42.17$]$ & $43.43(3.28)[36.71$ to 50.15$]$ & $36.26(3.94)[28 \cdot 16$ to $44 \cdot 36]$ & $26.46(3.82)$ [18.63 to 34.30$]$ \\
\hline Pain on visual analogue scale $\left(1^{\star}-100 \mathrm{~mm}\right)$ & $60 \cdot 89(4.07)[52 \cdot 52$ to $69 \cdot 25]$ & $56.61(3.19)$ [50.06 to 63.15$]$ & $53.78(4.37)[44.80$ to 62.76 ] & $39.89(4.97)[29.70$ to 50.06$]$ \\
\hline Sleep quality $\left(1-10^{\star}\right)$ & $4.70(0.38)[3.92$ to 5.49$]$ & $4.11(0.32)[3.45$ to 4.77$]$ & $4.85(0.43)[3.96$ to 5.74$]$ & $5.96(0.47)[5.00$ to 6.92$]$ \\
\hline Morning stiffness (minutes) & $82.04(13.11)$ [55.09 to 108.98$]$ & $57.86(11.80)[33.65$ to 82.07$]$ & $83.15(15.51)[51.26$ to 115.03$]$ & $40.89(10.64)[19.06$ to 62.73$]$ \\
\hline Patient's appreciation $\left(1-10^{\star}\right)$ & $4.59(0.26)[4.07$ to $5 \cdot 12]$ & $4.82(0.31)[4.18$ to 5.46$]$ & $5.07(0.37)[4.31$ to 5.84$]$ & $6.46(0.43)[5.58$ to 7.35$]$ \\
\hline Evaluating physician's appreciation $\left(1-10^{\star}\right)$ & $4.70(0.33)[4.03$ to 5.38$]$ & $5.21(0.32)[4.56$ to 5.87$]$ & $5.04(0.45)[4.12$ to 5.96$]$ & $7.00(0.41)[6.17$ to 7.83$]$ \\
\hline
\end{tabular}

^Figure marked with asterisk denotes best value. 


\begin{tabular}{|c|c|c|c|c|}
\hline & \multicolumn{2}{|c|}{$\mathrm{p}$ Value for intragroup changes ${ }^{\star}$} & \multicolumn{2}{|c|}{$\mathrm{p}$ Value for intergroup differences $\dagger$} \\
\hline & Control & Acupuncture & Before treatment & After treatment \\
\hline Pain threshold & $0 \cdot 6378$ & $0 \cdot 0027$ & $0 \cdot 8990$ & 0.0303 \\
\hline No of analgesic tablets during last week & 0.5379 & 0.0084 & 0.9931 & 0.0945 \\
\hline Regional pain score & 0.8192 & $0 \cdot 0000$ & $0 \cdot 1058$ & $0 \cdot 0570$ \\
\hline Pain on visual analogue scale & 0.0619 & 0.0020 & 0.2699 & 0.0246 \\
\hline Sleep quality & 0.9176 & 0.0004 & 0.2969 & 0.0782 \\
\hline Morning stiffness & $0 \cdot 8684$ & 0.0627 & $0 \cdot 1126$ & 0.0321 \\
\hline Patient's appreciation & 0.2360 & 0.0018 & 0.4353 & 0.0111 \\
\hline Evaluating physician's appreciation & 0.4080 & 0.0001 & 0.2266 & 0.0034 \\
\hline
\end{tabular}

*Wilcoxon matched pairs signed ranks test, two tailed.

tMann-Whitney $U$ and Wilcoxon rank sum W test, two tailed, corrected for ties.

difficult because the condition is mainly subjective with symptoms such as pain, stiffness, and sleep disturbance. No reliable outcome measure has been proposed. We therefore used several parameters in order to evaluate the different symptoms of fibromyalgia, as in trials evaluating tricyclic antidepressants. ${ }^{15} \mathrm{We}$ considered pain threshold to be the most important parameter as it is the most objective. After electroacupuncture the pain threshold improved by $70 \%$, and the difference compared with sham treatment was significant. The overall results in the active treatment group were similar to those in trials of antidepressants ${ }^{1516}$ and in the study by Lautenschläger et al, who also used acupuncture. ${ }^{10}$

In studies of the clinical effects of acupuncture a control procedure is difficult to devise. ${ }^{17}$ If needles are only attached to the skin, without penetration, ${ }^{18}$ then blindness of patients to the treatment may be difficult to assure and mock transcutaneous electrical nerve stimulation ${ }^{19}$ may have quite different psychological impact from real electroacupuncture. ${ }^{17}$ We believed that a control procedure should satisfy two conditions. Firstly, to assure blindness the treatment in the control group should induce sensations that accord with what patients would expect in real electroacupuncture-for example, insertion of needles and electric current. In this series patients who had had acupuncture previously were excluded and any contact between patients was avoided. On the other hand, the treatment should have the weakest possible effect.

Dry needling of muscle ${ }^{20}$ and a low frequency electric current (as in electroacupuncture) may have a local analgesic effect. We therefore inserted the needles, albeit less deeply, ${ }^{21}$ at sites distant from those used in acupuncture and used a weaker electric current. Indeed, with this procedure the patients seemed unaware of which group they were in. This was confirmed by almost identical drop out rates in the two groups and by the side effects reported in the control group. Exacerbation of symptoms during treatment was attributed by the patients to the effects of the needles and electric current.

The traditional points of acupuncture do not have a detectable anatomical substrate and their morphological basis remains controversial. Melzack et al thought that about two thirds of them corresponded to trigger points or to areas of referred pain. ${ }^{13}$ About a quarter of the points used for acupuncture lie above large nervous structures, so that needles may reach the perineum and function as transcutaneous electrodes. The analgesic effect of stimulating such structures has been well documented. ${ }^{22}{ }^{23} \mathrm{At}$ the point located over the first dorsal interosseous muscle the needle penetrates the skin (radial nerve), reaches the muscle (ulnar nerve), and contacts sensitive branches of the median nerve and might therefore activate a diverse number of nerve fibres. The relation between spatial summation of neural activation and analgesic effect of electroacupuncture was proposed by Toda and Ischioka. ${ }^{24}$

These observations suggest that the site and depth of insertion may be relevant. Unfortunately, there is poor experimental evidence about the importance of these two parameters for the efficacy of acupuncture. No more is known about the specific action of the different points as postulated by the traditional acupuncture rules. In our study we used the traditional acupuncture points as sites of insertion, the choice of the points being mainly empirical, with special attention to areas that had been used in analgesia experiments. 35720232428 We avoided inserting needles into the tender points because we had often observed a worsening of pain when tender points were stimulated by electroacupuncture. The pathophysiological significance of this observation remains unclear.

The exact mode of action of acupuncture remains unknown but there are some consistent data in favour of a neurohumoral mechanism..$^{3-61225}$ Most workers support the hypothesis that electroacupuncture may activate some endogenous pain control mechanisms, ${ }^{20-28}$ such as the opioid mediated analgesia system $^{29-31}$ or serotoninergic pathways. ${ }^{32}$ The analgesic effect can occur at the segmental level, by heterotopic stimulation, especially through the activation of the brain stem to spinal cord network, ${ }^{26}$ or via systemic release of peptides by the adrenals. ${ }^{33}$ The activation of some endogenous pain modulating systems via peripheral heterotopic stimulation was well documented by Le Bars et al in their studies on diffuse noxious inhibitory controls, which may share some common mechanisms with electroacupuncture. ${ }^{34}$

The results of this study suggest that electroacupuncture has an objective beneficial effect in fibromyalgia. Whether electroacupuncture might be useful in long term management was not examined and needs further study. The findings of Waylonis ${ }^{9}$ and of Lautenschläger et $a l^{10}$ and unpublished data suggest that the effect of electroacupuncture outlasts the duration of treatment by several weeks in most patients. The advantage of acupuncture over antidepressants or anti-inflammatory drugs often used in fibromyalgia is the low rate of side effects when administered by a physician with adequate technical skill and under proper aseptic conditions. Acupuncture and especially electroacupuncture might become a useful adjunct in the treatment of fibromyalgia and other chronic osteoarticular pain syndromes.

We thank Ms Bernadette Mermillod, Centre Informatique Hospitalière, University Hospital, Geneva, for advice during the planning of the study. Statistical analysis was by A Clemence, of the University of Clermont-Ferrand, France, using the SPSS statistical package for IBM personal computers.

1 Bennett RM. Fibrositis. In: Kelley WN, Harris ED, Ruddy S, Sledge CB, eds Textbook of rheumatology. Philadelphia: W B Saunders, 1989:541-53

2 Buckelew SP. Fibromyalgia: a rehabilitation approach. Am f Phys Med Rehabil 1 989;68:37-42.

3 He L, Dong WQ, Wang M. Effects of jontophoretic etorphine and naloxone, and electroacupuncture on nociceptive responses from thalamic neurons in rabbits. Pain 1991;44:89-95.

4 Pommeranz B, Cheng R. Suppression of noxious responses in single neuron of cat spinal cord by electroacupuncture and its reversal by the opiate

5 Mayer DJ, Price DD, Rafii A. Antagonism of acupuncture analgesia in man by the narcotic antagonist naloxone. Brain Res 1977;121:368-72.

6 Price DD, Rafii A, Watkins LR, Buckingham B. A psychophysical analysis of acupuncture analgesia. Pain 1984;19:27-42.

7 Willer JC, Roby A, Boulu P, Boureau F. Comparative effects of electroacupuncture and transcutaneous nerve stimulation on the human blink reflex Pain 1982;14:267-78.

8 Riet GT, Kleijnen J, Knipschild P. Acupuncture and chronic pain: a criteriabased meta-analysis. f Clin Epidemiol 1990;43:1191-9.

9 Waylonis GW. Long term follow-up on patients with fibrositis treated with acupuncture. Ohio State Med f 1977;73:299-302.

10 Lautenschläger J, Schnorrenberger CC, Muller W. Akupunktur bei generalisierter Tendomyopathie (Fibromyalgie-Syndrom). Dtsch Zschr Akup 1989; 6:122-8.

11 American College of Rheumatology. Criteria for the classification of fibromyalgia. Arthritis Rheum 1990;33:160-71.

12 Macdonald AJR. Acupuncture analgesia and therapy. In: Wall PD, Meizack R, eds. Textbook of pain. Edinburgh: Churchill Livingstone, 1989;906-19.

13 Melzack R, Stillwell M, Fox E. Trigger points and acupuncture points for pain: correlation and implications. Pain 1977;3:2-23.

14 Reeves JI, Jaeger B, Graff-Radford SB. Reliability of the pressure algometer as a measure of myofascial trigger point sensitivity. Pain 1986;24:313-21 
15 Bennett RM, Gatter RA, Campbell SM, Andrews RP, Clark SR, Scarola JA. A comparison of cyclobenzaprine and placebo in the management of fibrositis. Arthritis Rheum 1988:31:1535-42.

16 Carette S, McCain A, Fam AG. Evaluation of amitriptyline in primary fibrositis. Arthritis Rheum 1986;29:655-9.

17 Vincent CA, Richardson PH. The evaluation of therapeutic acupuncture: concepts and methods. Pain 1986;24:1-13.

8 Moore ME, Berk SN. Acupuncture for chronic shoulder pain. Ann Intern Med 1976;84:381-4.

19 Lehmann T, Russel DW, Spratt KF, Colby H, Liu YK, Fairchild ML, et al. Efficacy of electroacupuncture and TENS in the rehabilitation of chronic low back pain patients. Pain 1986;26:277-90.

20 Lewit K. The needle effect in the relief of myofascial pain. Pain 1979;6:83-90.

21 Hansen PE, Hansen JH Acupuncture treatment of chronic facial pain: a controlled cross-over trial. Headache 1983:23:66-9.

22 Lee $\mathrm{KH}$, Chung JM, Willis WD. Inhibition of primate spinothalamic tract cells by TENS. F Neurosurg 1985:62.276-87.

23 Paik KS, Nam SC, Chung JM. Differential inhibition produced by peripheral conditioning stimulation on noxious mechnical and thermal responses of conditioning stimulation on noxious mechnical and thermal responses

24 Toda $\mathrm{K}$, Ischioka $M$. Electroacupuncture: relations between forelimb afferent impulses and suppression of jaw-opening reflex in the rat. Exp Neurol 1978;61:465-70

25 Han JS. Central neurotransmitters and acupuncture analgesia. In: Pomeranz B, Stux G, eds. Scientific bases of acupuncture. Berlin: Springer Verlag, 1989:7-33.
26 Fields HL, Basbaum AI. Endogenous pain control mechanisms. In: Wall PD, Melzack R, eds. Textbook of pain. Edinburgh: Churchill Livingstone, 1989:206-17.

27 Eadie MJ. Acupuncture and the relief of pain. Med $\mathcal{F}$ Aust 1990;153:180-1

28 Cheng RSS. Neurophysiology of electroacupuncture analgesia. In: Pomeran B, Stux G, eds. Scientific bases of acupuncture. Berlin: Springer Verlag, 1989:119-36

29 Ho WKK, When HL. Opioid-like activity in the cerebrospinal fluid of pain patients treated by electroacupuncture. Neuropharmacology 1989;28:961-6.

30 Bing Z, Cesselin F, Clot AM, Hamon M, Le Bars D. Acupuncture-like stimulation induces a heterosegmental release of Met-enkephalin-like material in the rat spinal cord. Pain 1991;47:71-7

31 Clement-Jones V, McLoughlin L, Tomlin S, Besser GM, Rees LH, Wen HL. Increased B-endorphin but not met-enkephalin levels in human cerebrospinal fluid after acupuncture for recurrent pain. Lancet 1980;ii:946-8.

32 Han JS. Electroacupuncture: an alternative to antidepressants for treating affective diseases? Int 7 Neurosci 1986;29:79-92.

33 Sandford Kiser R, Gatchel RJ, Bhatia K, Khatami M, Huang XY, Altshuler $\mathrm{KZ}$. Acupuncture relief of chronic pain syndrome correlates with increased plasma metenkephalin concentrations. Lancet 1983;ii: 1394-6.

34 Le Bars D, Willer JC, De Broucker T, Villanueva L. Neurophysiological mechanisms involved in the pain-relieving effects of counter-irritation and related techniques including acupuncture. In: Pomeranz B, Stux G, eds. Scientific bases of acupuncture. Berlin: Springer Verlag, 1989:79-112.

(Accepted 21 September 1992)

\title{
Tobacco and myocardial infarction: is snuff less dangerous than cigarettes?
}

\author{
F Huhtasaari, K Asplund, V Lundberg, B Stegmayr, P O Wester
}

Abstract

Objective-To estimate the risk of myocardial infarction in snuff users, cigarette smokers, and nontobacco users in northern Sweden, where using snuff is traditional.

Design-Case-control study.

Setting-Northern Sweden.

Subjects-All 35-64 year old men who had had a first myocardial infarction and a population based sample of 35-64 year old men who had not had an infarction in the same geographical area.

Main outcome measure-Tobacco consumption (regular snuff dipping, regular cigarette smoking, non-tobacco use) and risk of acute myocardial infarction.

Results-59 of $585(10 \%)$ patients who had a first myocardial infarction and 87 of $589(15 \%)$ randomly selected men without myocardial infarction were non-smokers who used snuff daily. The age adjusted odds ratio for myocardial infarction was $0.89(95 \%$ confidence interval 0.62 to 1.29 ) for exposure to snuff and $1.87(1.40$ to 2.48$)$ for cigarette smoking compared with non-tobacco users, showing an increased risk in smokers but not in snuff dippers. Regular cigarette smokers had a significantly higher risk of myocardial infarction than regular snuff dippers (age adjusted odds ratio $2 \cdot 09 ; 1.39$ to $3 \cdot 15$ ). Smoking, but not snuff dipping, predicted myocardial infarction in a multiple logistic regression model that included age and level of education.

Conclusions-In middle aged men snuff dipping is associated with a lower risk of myocardial infarction than cigarette smoking.

University Hospital,

S-901 85 Umeå, Sweden

K Asplund, senior lecturer

B Stegmayr, research assistant

P O Wester, professor

Department of Medicine,

Kalix Hospital, S-952-82

Kalix, Sweden

V Lundberg, research

assistant

Correspondence to:

Dr Asplund.

BMF 1992;305:1252-6 advertise cigarettes; whereas there is a massive documentation of the many detrimental effects of smoking tobacco, knowledge about the health effects of smokeless tobacco is limited. ${ }^{78}$

Cigarette smoking kills by increasing the risk of various atherothrombotic disorders, in particular myocardial infarction. ${ }^{9}$ Although smokeless tobacco has been implicated in the pathogenesis of circulatory disorders, including coronary artery disease, the evidence is mostly circumstantial. ${ }^{78} \mathrm{We}$ therefore estimated the risk of myocardial infarction among snuff dippers in northern Sweden, where the use of snuff is traditional in middle aged men-a group at considerable risk of myocardial infarction.

\section{Subjects and methods}

This case-control study was performed within the framework of the northern Sweden MONICA project (multinational monitoring of trends and determinants in cardiovascular disease). The World Health Organisation MONICA project tests the hypothesis that changes over time in commonly recognised cardiovascular risk factors such as hypercholesterolaemia, hypertension, and cigarette smoking affect incidence and mortality in acute myocardial infarction and stroke. ${ }^{10}$ Thirty nine populations in 26 countries are being followed up for 10 years. The northern Sweden project covers the two northernmost provinces of Sweden, Norrbotten and Västerbotten, with a total population of 510000 living in an area of $154000 \mathrm{~km}^{2}$.

We compared the pattern of tobacco consumption in patients with acute myocardial infarction with that of participants in a MONICA population survey of cardiovascular risk factors. Cases and controls were from the same population of 35-64 year old men. The prevalence of snuff dipping among women was too low to permit meaningful analysis.

During the period April 1989 to April 1991, 629 men aged 35-64 in northern Sweden had their first acute myocardial infarction. Case finding followed the structured MONICA procedures ${ }^{10}$ and was based on reports from general practitioners and the nine acute care hospitals in the area, checks of computerised discharge registers, and screening of death certificates of all subjects who died in the two provinces. Uniform 